\title{
Manejo del paciente pediátrico en el consultorio dental
}

Dr. Walter Aníbal López Álvarez ${ }^{1}$

Dr. Roberto Gordillo Castillo ${ }^{2}$ Lcda. María Sofía Álvarez ${ }^{3}$

\section{Resumen}

El propósito de este artículo es introducir a los lectores al conocimiento de las alternativas que existen sobre el manejo adecuado del comportamiento y uso de las diferentes alternativas en el tratamiento dental del paciente pediátrico. El odontropediatra enfrenta un reto muy interesante con cada uno de los niños que llegan a su clínica, ya que la cultura de higiene bucal y prevención de la misma, aún no está presente en la población guatemalteca. Las ventajas del tratamiento dental bajo anestesia general son muchas: los tratamientos requeridos se realizan en una sola sesión, en un entorno seguro. Además, durante la anestesia general (AG) se asegura un control efectivo del dolor. Las desventajas también existen: todos los agentes anestésicos están asociados con cierto riesgo para el paciente. Es por esto que se requiere la presencia de personal altamente calificado en AG y el monitoreo continuo del paciente.

Palabras clave: Odontopediatra, sedación, anestesia general (AG)

\section{Abstract}

The purpose of this article is to introduce our readers to the alternatives that exist for the proper management of the behavior and the use of different types of sedation for dental treatments. The pediatric dentist faces a very interesting challenge with each of these children who come to the office not having the cultural background of oral hygiene and prevention, not yet present on the Guatemalan population. The advantages of dental treatment under General Anesthesia (GA) are many: the required treatments are performed in a single session and in a safe environment. In addition, effective pain control is ensured during GA. You do not need child cooperation as a requirement for the treatment. However, disadvantages also exist. All anesthetic agents are associated with risk factors affecting to the patient. There are morbidity and mortality reports that demonstrate this. The presence of highly qualified personnel during the procedure (GA) and the continuous monitoring of the patient is required.

Keywords: pediatric dentist, sedation, general anesthesia (GA)

\footnotetext{
${ }^{1}$ Dentista pediátrico por la University of Pittsburgh (Clínica Dental Waly, S. A.).

${ }^{2}$ Doctor anestesiólogo por la Universidad de San Carlos de Guatemala.

${ }^{3}$ Licenciada en Psicología Clínica por la Universidad Rafael Landívar.
} 


\section{Introducción}

El manejo del comportamiento es cuando el equipo dental efectúa eficientemente un tratamiento y al mismo tiempo crea una actitud positiva hacia los procedimientos odontológicos en el niño. Aunque la operatoria dental sea perfecta, la cita fue un fracaso si el paciente llora. Existen dos tipos de manejo en un paciente:

- Manejo no-farmacológico

- Manejo farmacológico

Trabajar profesionalmente con niños y que ellos logren aceptar e instalar de manera adecuada cuidados y hábitos, requiere conocimiento del desarrollo cognitivo y psicológico; esto con el fin de lograr medidas preventivas en el paciente y como profesional, comprender el comportamiento del individuo para la selección de un tratamiento conductual adecuado y efectivo (1).

En cuanto al tema de higiene bucal, el odontopediatra, por su parte, enfrenta un reto muy interesante con cada uno de estos niños que llegan a su clínica, ya que, la cultura de higiene bucal y prevención de la misma, aún no está instalada culturalmente en la mayoría de guatemaltecos.

\section{Miedo y ansiedad en el paciente infantil}

Trabajar con niños es diferente a trabajar con adultos, no todos son iguales, y la experiencia clínica es un factor importante. Uno de los aspectos más importantes en odontopediatría es el control de conducta, pues sin cooperación por parte del niño no es posible realizar con éxito su tratamiento dental (2).

\section{Técnicas no farmacológicas}

\section{Técnicas de comunicación:}

- Comunicación verbal

- Comunicación no verbal

- Decir, mostrar, hacer

- Control de voz

\section{Técnicas de modificación de la conducta:}

- Desensibilización

- Imitación

\section{Técnicas de limitación:}

- Abrebocas, bloqueo de mordida

- Inmovilización medica por parte del equipo dental

\section{Técnicas prohibidas:}

- Mano sobre la boca

\section{Otras técnicas:}

Cada vez son más numerosos los padres que rechazan las técnicas clásicas de control de la conducta del niño (inmovilización), a pesar de ser efectivas resultan inaceptables por parte de la mayoría de los padres. 
Ante esta situación la profesión se ha visto obligada a limitar la utilización de ciertas técnicas eficaces pero consideradas inaceptables por los padres, para potenciar otras, como distracción, relajación, magia, hipnosis, acupuntura y psicólogos infantiles.

\section{Técnicas farmacológicas Indicaciones de la sedación}

- Pacientes ansiosos y con miedo en los cuales las técnicas de modificación conductual no hayan sido exitosas.

- Pacientes que no puedan cooperar debido a una escasa maduración psicológica o emocional, o en pacientes con discapacidad física, mental o médica.

- Pacientes en los que el procedimiento de sedación reduzca el riesgo médico.

- Pacientes muy pequeños que sean poco cooperadores.

- Pacientes con necesidad de tratamientos muy extensos.

- Pacientes que requieran viajar desde muy lejos para recibir tratamiento.

\section{Tipos de sedación:}

- Sedación consciente leve (ansiolisis)

- Sedación consciente moderada (sedación consciente o sedación / analgésica)

- Sedación profunda

- Anestesia general
Vías de administración:

- Oral

- Inhalada

- Intravenosa

- Fármacos utilizados

o Óxido nitroso

o Sevoflurano

- Fármacos hipnóticos por vía intravenosa:
o Metohexital
o Midazolam
o Ketamina
o Fentanilo

En los últimos años, el uso de la sedación para procedimientos odontológicos ha sido más racional basados en la evidencia científica de las últimas décadas, el beneficio mayor es para pacientes niños y pacientes adultos con fobia a procedimientos dentales.

La administración de anestesia o sedación en niños en lugares distintos a los quirófanos, específicamente en el consultorio dental, requiere una planificación previa y una organización cuidadosa por parte de los profesionales que brindaran la atención al niño. Esto inicia desde la evaluación oral preoperatoria y la evaluación integral de la salud del paciente, y una estrecha colaboración entre los proveedores de la atención dental y de la AG.

El tratamiento dental integral bajo AG es en la actualidad un procedimiento seguro, siempre y cuando se cumplan con los estándares de calidad en el servicio. Además de los criterios para la selección de casos de anestesia general dental, se deben considerar los siguientes factores: a) factores propios del paciente: la edad, capacidad para cooperar, ansiedad, enfermedades o lesiones concomitantes; b) factores propios del equipo proveedor del servicio: el nivel de entrenamiento del anestesiólogo y del odontopediatra, disponibilidad de todos los suministros de asistencia, monitorización, reanimación y recuperación; y c) factores propios de la intervención: duración del procedimiento, colocación del paciente, dolor previsto, las instrucciones pre y postoperatorias a los padres y el consentimiento informado (6).

Los odontopediatras tratan a los niños y utilizan las técnicas convencionales de manejo del comportamiento. Sin embargo, hay un grupo de niños que no es posible manejar a través de estos métodos $(3,5,7)$.

En algunos casos, la AG dental es el modo de tratamiento más práctico y económico (4, 5). Según la Academia Americana de Odontología Pediátrica (AAPD, por sus siglas en inglés), una cierta población de pacientes que no puede tolerar el tratamiento dental de rutina solo puede ser tratada bajo AG. Los pacientes pediátricos con una edad muy temprana, o aquellos que padecen inmadurez o discapacidad física, mental, cognitiva o emocional, o aquellos con ansiedad extrema que necesitan rehabilitación extensa se tratan con AG. Estos niños no son candidatos adecuados para los tratamientos convencionales 
en el consultorio y son tratados de manera más segura y efectiva bajo AG. La mayoría de los candidatos a la AG dental son niños que padecen un problema de salud prevalente, caries en la primera infancia (ECC) y, por lo demás, están sanos $(3,5,7)$.

La evaluación preoperatoria le permite al anestesiólogo elaborar la estrategia y tácticas correctas para garantizar la adecuada conducción anestésica durante el procedimiento dental. El paciente pediátrico posee características fisiológicas relacionadas con la edad que lo diferencian del adulto, ello determina un riesgo diferente ante el manejo anestésico $(3,5,6)$.

La valoración preoperatoria (días antes del procedimiento electivo $u$ horas o minutos antes en los procedimientos de urgencia), es la manera más efectiva de reducir el riesgo anestésico. Entre los aspectos a valorar por el anestesiólogo están: antecedentes del nacimiento, malformaciones congénitas, trastornos hematológicos, trastornos cardíacos o respiratorios, presencia de infección respiratoria aguda, horas de ayuno. Además, es imprescindible advertir a los padres sobre posibles efectos secundarios y complicaciones que conlleva el procedimiento (6). La mayoría de los pacientes pediátricos reportan quejas después del procedimiento de AG dental con diferente gravedad. Dolor dental, dificultad para comer, sangrado nasal, malestar en la garganta, malestar nasal, alteración del sueño, debilidad, somnolencia, deshidratación, fiebre, náuseas, y estreñimiento. El dolor postoperatorio es la queja más común reportada $(6,8,9,10)$. En el estudio de Cantekin, efectuado en niños sanos de cuatro a diez años de edad, la prevalencia de molestias postoperatorios fue del $59 \%$ (8). El estudio de Farsi efectuado en niños sanos entre uno y trece años de edad, reportó un $99 \%$ de problemas postoperatorios (la mayoría de mediana severidad) durante el primer día postoperatorio, con un drástico descenso a $33 \%$ de las molestias al tercer día (10).

El ayuno preoperatorio ha sido un tema de interés por parte de los anestesiólogos, con la finalidad de prevenir el vómito y regurgitación durante la inducción anestésica, sobre todo en pacientes con «estómago lleno» y que constituye un peligro de aspiración bronquial. Asimismo, existe correlación entre el antecedente de infección respiratoria aguda (IRA) y complicaciones respiratorias cuando se administra anestesia general (6).

Las instrucciones postoperatorias son muy importantes. Los niños deben ser supervisados durante las primeras veinticuatro horas después del procedimiento. Es necesaria una instrucción escrita, para que los padres estén informados sobre cualquier síntoma experimentado y la posible secuencia de eventos. Las instrucciones deben ser claras y deberán incluir el manejo de la tolerancia de líquidos y el uso de analgésicos. La actividad del niño deberá ser restringida solo a interiores, porque el equilibrio y la coordinación pueden tardar varias horas en volver al estado normal $(3,5,6,7)$.
Durante la rehabilitación dental integral con AG, un trabajo en equipo efectivo es la clave para llevar a cabo servicios dentales óptimos. Los individuos entrenados, experimentados y capacitados en el equipo de operación son críticos para evitar cualquier riesgo y al mismo tiempo brindar la atención más adecuada y de alta calidad. Mantener las funciones fisiológicas y la respiración adecuada del paciente infantil que se somete a $A G$ es el papel del anestesiólogo dental y sus asistentes. El especialista revisa continuamente los signos vitales durante la operación $(3,5,6,7)$. El niño debe ser vigilado hasta que retorne la conciencia. Durante la recuperación el paciente debe ser vigilado cuidadosamente. Además, los pacientes con problemas médicos importantes, deben ser atendidos por un equipo médico que incluya un pediatra $(3,7)$.

Los odontopediatras, además de realizar el tratamiento convencional mediante la aplicación de técnicas de manejo de la conducta, deberán estar capacitados para realizar el tratamiento con AG. En este sentido, se hace hincapié en la importancia de brindar los tratamientos más duraderos y exitosos para evitar otra AG y brindar al niño una alta calidad de vida relacionada con la salud oral $(3,4,5,7)$.

El tratamiento dental bajo AG tiene la ventaja de que se realiza en una sola sesión y en un entorno seguro. Además, durante la $A G$ se asegura un control efectivo del dolor. Se requiere de muy poca o ninguna colaboración del niño que va a 
ser sometido al tratamiento. $Y$ el tratamiento dental bajo AG es más económico y la calidad y durabilidad suelen ser superiores a los tratamientos convencionales $(3,4,5,6,7)$.

Sin embargo, las desventajas también existen: todos los agentes anestésicos están asociados con cierto riesgo para el paciente. La bibliografía consultada nos habla de la morbilidad encontrada en esta clase de procedimientos $(8,9$, $10,11)$. Lee y colaboradores, analizan las muertes relacionadas

al tratamiento dental en el

periodo 1980-2011, en los

Estados Unidos. Aunque los

alcances de este estudio no

llegan a establecer la incidencia

y prevalencia de la morbilidad y mortalidad global, si nos indica que el $50 \%$ de las muertes es en niños entre 2 y 5 años de edad y que las complicaciones son más frecuentes cuando los proveedores son menos especializados y en el entorno de la oficina del odontólogo más que en los hospitales (12).

\section{Referencias}

1. Castillo I, Ledo H, Ramos A. Psicoterapia Conductual en niños: estrategia terapéutica de primer orden. Norte de salud mental. 2012;10(43):30-36.

2. Appukuttan DP. Strateies to manage patients with dental anxiety and dental phobia: literatura review. Clin Cosmet Investig Dent 2016;8:35-50.

3. Jankauskiene B, Virtanen Ji, Kubilius R, Narbutaite J: Tratamiento con anestesia general dental en niños menores de 6 años en Lituania. Medicina. Kaunas. 2013;49(9):403-408.

4. Lee JY, Vann WJ, Roberts MW. Un análisis de costos del tratamiento de pacientes dentales pediátricos con anestesia general versus sedación consciente. Anestes Prog. 2001;48(3):82-88.

5. Lee PY, Chou MY, Chen YL, Chen LP, Wang CJ, Huang WH. Tratamiento dental integral bajo anestesia general en niños sanos y discapacitados. Chang Gung Med J. 2009;32(6):636-642.

6. Bell CH, Kain ZN, Hughes C. «Valoración preoperatoria», en Manual de anestesia pediátrica. Barcelona: Harcourt, 2. ${ }^{\text {a }}$ ed. 1998; pp. 3-17.

7. Academia Americana de Odontología Pediátrica Comité Ad Hoc sobre Sedación y Anestesia Academia Americana de Odontología Pediátrica, Consejo de Asuntos Clínicos: Política sobre el uso de sedación profunda y anestesia general en el consultorio dental pediátrico. Pediatr Dent. 2008-2009;30(7):66-67.

8. Cantekin K, Yildirim MD, Delikan E, Cetin S: Molestias postoperatorias de la rehabilitación dental bajo anestesia general. Pak J Med Sci. 2014;30(4):784-788.

9. Needleman, HL., Harpavat S, Wu S, Allred EN, Berde C. Dolor postoperatorio y otras secuelas de rehabilitaciones dentales realizadas en niños bajo anestesia general. Pediatr Dent. 2008;30:11-121.

10. Farsi N, Ba'akdah R, Boker A, Almushayt A. Complicaciones postoperatorias del procedimiento de anestesia general dental pediátrica en hospitales de Jeddah, Arabia Saudita. BMC Oral Health. 2009;9-16. doi: 10.1186/1472-6831-9-6.

11. Atan S, Ashley P, Gilthorpe MS, Scheer B, Mason C, Roberts G. Morbilidad después del tratamiento dental de niños bajo anestesia general de intubación en una unidad de día. Int J Paediatr Dent. 2004;14(1):9-16.

12. Lee HH, Milgrom P, Starks H, Burke W. Tendencias en muertes asociadas con la sedación dental pediátrica y la anestesia general. Paediatr Anaesth. 2013;23(8):741-746. doi: 10.1111/pan.12210. 


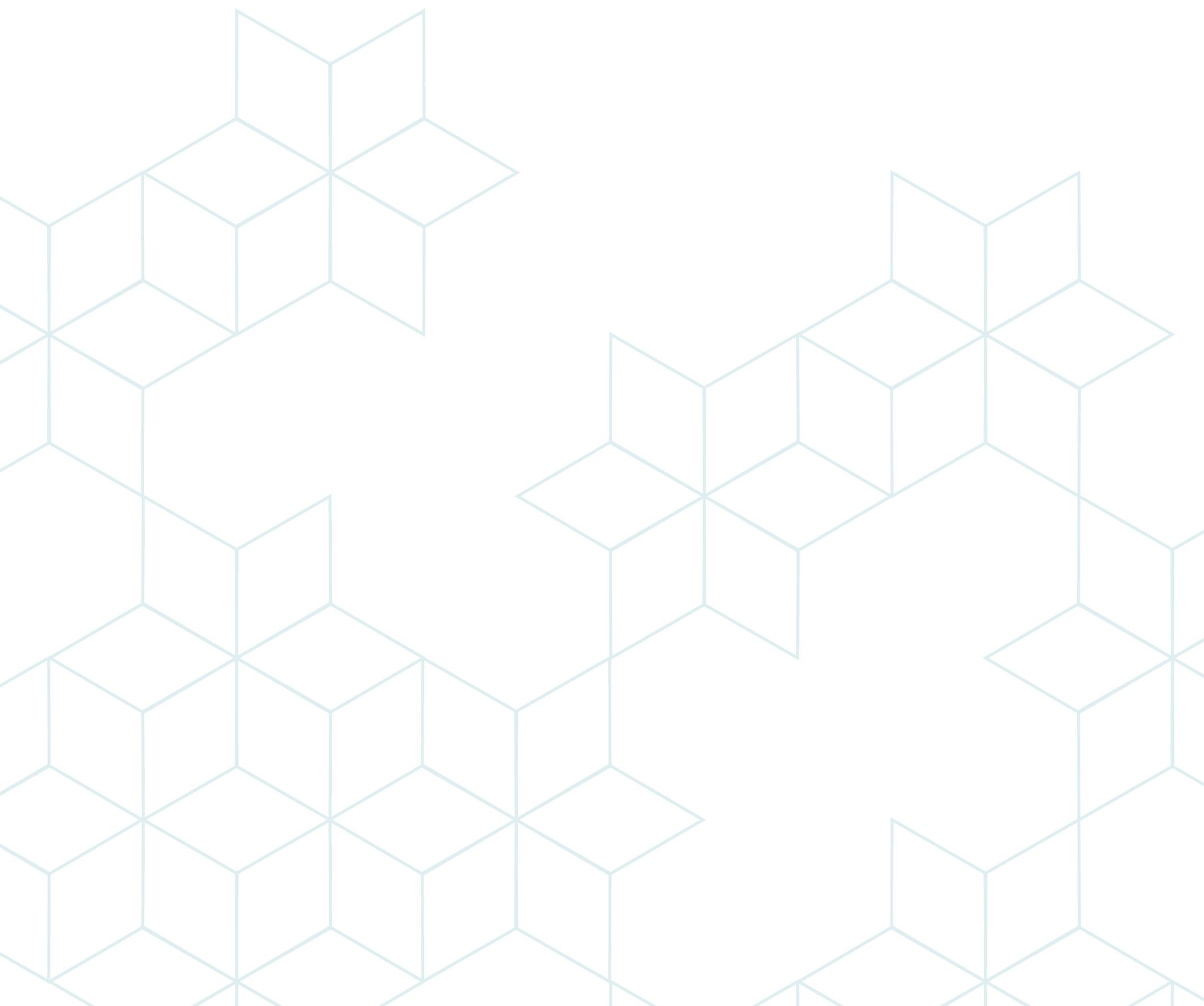

